Anales de Geografía de la Universidad Complutense ISSN: 0211-9803

https://dx.doi.org/10.5209/aguc.79343

\title{
La producción del espacio de acumulación y representación cultural en Madrid (2000-2010)
}

\author{
Daniel Morcillo Álvarez ${ }^{1}$ \\ Recibido: 6 de mayo del 2021 / Enviado a evaluar: 20 de junio del 2021 / Aceptado: 10 de diciembre del 2021
}

\begin{abstract}
Resumen. En las últimas décadas se ha considerado la cultura desde planos diversos, y vista como mecanismo de identificación social se ha expandido por los espacios centrales de las ciudades dando lugar a un modelo de regeneración urbana, que produce nuevos espacios de representación. En Madrid, las inversiones públicas realizadas han permitido la reutilización de edificios y lugares, transformando con ello los usos, la imagen y los cuadros de representación social a los que estaban asociados. El artículo busca el origen de esta realidad en los años de máxima expansión inmobiliaria y de circulación de capital, algo que contribuiría a una transformación del capital simbólico en el que se apoya. A partir del análisis de la expansión geográfica de los espacios culturales y en las inversiones asociadas en la ciudad de Madrid, se ha pretendido reconocer los vínculos entre el proceso de producción-acumulación de capital y los de producción de espacio.
\end{abstract}

Palabras clave: Morfología, cultura, producción de espacio, posición social, centralidad urbana, inversión pública.

\section{[en] The production of the space of accumulation and cultural representation in Madrid (2000-2010)}

\begin{abstract}
Culture was considered in last decades along several points of view, and considering it as an instrument of social identification, it was spread along the urban centers of the cities in which was growing a new kind of urban regeneration and the production of new spaces for social rise. In Madrid, public investments have allowed the re-use of buildings and urban structures that have changed the uses, images and social representation that they were associated. This paper looks for the origin of this situation in those times of real state expansion, in that moment that capital was flowing in the markets to contribute to the symbolic capital transformation. From de analysis of geographical expansion of cultural spaces and their associated investments in Madrid, the paper has wanted to find the relationship between the capital production-accumulation and the space production.
\end{abstract}

Keywords: Morphology, culture, space production, social position, urban center, public investment.

1 Departamento de Urbanística y Ordenación del Territorio de la Escuela Técnica Superior de Arquitectura de Madrid. Universidad Politécnica de Madrid.

E-mail: daniel.morcillo@upm.es 


\title{
[fr] La production de l'espace d'accumulation et de représentation culturelle à Madrid (2000-2010)
}

\begin{abstract}
Résumé. Au cours des dernières décennies, la culture a été considérée à différents niveaux et sa vision en tant que méthode d'identification sociale s'est étendue aux espaces centraux des villes.. Ce processus a produit un modèle de régénération urbain avec nouvelles espaces de représentation sociale. À Madrid, les investissements publics ont permis la réutilisation des bâtiments et des lieux qui ont représenté la transformation des usages, de l'image et des cadres de représentation sociale qui leur sont associés. L'article cherche l'origine de cette réalité à l'époque de la plus haute expansion de l'urbanisation et de la circulation du capital économique, conditions de la transformation du capital symbolique associé au capital économique. L'analyse de l'expansion des lieux culturels et de leurs investissements publics à Madrid, on a prétendu reconnaître les liens entre les processus de production-accumulation du capital et la production de l'espace.
\end{abstract}

Mots Clès: Morphologie, culture, production de l'espace, position sociale, centre urbain, investissement publique.

Cómo citar. Morcillo Álvarez, D. (2021): La producción del espacio de acumulación y representación cultural en Madrid (2000-2010). Anales de Geografía de la Universidad Complutense, 41(2), 409-429.

\section{Sumario.}

1. Introducción. 2. Aspectos morfológicos y producción espacial en Madrid. 3. Proceso de acumulación en el espacio de centralidad de Madrid: la Almendra Central. 4. El uso cultural en la Almendra Central. 5. Inversiones culturales en la expansión de la centralidad. 6. Conclusiones. 7. Referencias bibliográficas.

\section{Introducción}

Tras la larga noche de la crisis económica de la primera década del siglo XXI, cuyos ecos resuenan hoy en la actual crisis sanitaria, surgen preguntas cuyas respuestas parecen tener origen en los años previos a la quiebra de Lheman Brother. Es recurrente acudir a conceptos que parecen surgir como novedad, pero, en el fondo, se arraigan en las formas de producción del espacio llevadas a cabo en las décadas precedentes a la crisis y que se consolidaron en la larga última década. Las formas de crecimiento urbano bajo el neoliberalismo se basaron en la expansión del mercado de vivienda que, ambicionando la producción ilimitada de capital, produjo un espacio diferenciado, fuertemente desigual y socialmente segregado (Méndez, 2012). En la competencia entre espacios subyacía la idea de estatus urbano, en una pugna por adquirir posición social traducida en una mayor intensificación de la localización de usos y actividades donde se reflejara el estatus espacial y a partir suyo, el de clase.

Para la consolidación de este modelo fueron necesarias fuertes inversiones que lograron una expansión tal de la urbanización que generó el espejismo de la reproducción ilimitada de capital, y convirtió a las ciudades, tal y como sugiere Harvey (Harvey, 2013), en el origen de la crisis económica. Sin embargo, aún queda por demostrar si en estos años se estaban estableciendo las bases que llevaran a un cambio de modelo urbano, y los fuertes vínculos entre crisis y producción de un espacio social adecuado al modelo. El análisis de las inversiones realizadas en los 
centros urbanos nos hace pensar que en los años que precedieron a la crisis se estaba dando ya, de facto, un cambio de ciclo, con una importante intensificación de la acumulación de capital público para minimizar los riesgos del capital privado, pero también un cambio en el espacio social, intensificando las desigualdades y la desposesión de las capas populares asalariadas. El reflejo de los campos en los que se ha producido la inversión pública en estos años previos a la crisis nos da pistas de hacia dónde caminaban las ciudades y qué modelo se estaba adoptando. Este es el caso de la inversión en infraestructuras culturales, a las que Neil Smith (2010) otorga un papel relevante para la expansión liberal. La cultura es uno de los factores que determinan el auge de un lugar frente a otro, por lo que las ciudades han dado peso a su desarrollo de la cultura para posicionarse mejor en la atracción de capital.

Madrid no ha sido ajena a ello. Tomada como una decisión estratégica, la acumulación de capital simbólico en torno a infraestructuras culturales de diferente entidad y características, ha permitido recuperar arquitecturas abandonadas, reconfigurar espacios históricos y transformar áreas urbanas, con el objetivo último de situarla entre las principales ciudades de atracción del turismo cultural de Europa y como apoyo a la atracción de capital internacional (Subdirección General de Análisis Socioeconómico, 2018), contribuyendo con ello, a la producción de una ciudad global capaz de atraer a millones de personas dedicadas al consumo, al ocio y, en definitiva, a la actividad recreativa.

De esta manera, el artículo se fundamenta en un método estadístico cuyos resultados son representados en una serie de mapas que muestran la evolución del número y localización de los locales de actividad cultural. En este sentido, se toman los datos del Directorio de Unidades de Actividades Económicas con la finalidad de establecer las series estadísticas que reflejen en qué lugar de la ciudad se han ido acumulando las actividades culturales, comparándolas con los espacios de acumulación de las inversiones públicas. Ello nos da una muestra representativa de su expansión como instrumento de producción de espacios de representación. Asimismo, la inversión pública en infraestructuras culturales nos da una idea de la relevancia de estas en la producción espacial neoliberal.

En base a estos datos, podemos encontrar una gran acumulación de locales culturales en las áreas centrales, mientras que éstos son de menor entidad y carácter local en las periferias de la ciudad. La inversión realizada en la ciudad es, pues desigual, y un análisis de esta en los años de máxima expansión neoliberal (entre los años 2000 y 2008), nos desvela la fuerte inversión pública en infraestructuras culturales localizadas en el centro. Es así cómo algunas representaciones urbanas, surgidas en los últimos años, parecen derivadas más que de la turistificación o gentrificación, de la competencia entre lugares por alcanzar un estatus reflejo del cuadro de representación social determinante en la fase neoliberal. 


\section{Aspectos morfológicos y producción espacial en Madrid}

La sociedad neoliberal debe sus costumbres a estar embebida de un «sentido común económico» (Bourdieu, 2003), es decir, a unas estructuras sociales y cognitivas propias a los modos de producción neoliberales, lo que supone que todos los aspectos que le dan forma estén supeditados a este, incluido el espacio. Si bien desde el siglo XIX parecía que la burguesía no requería de espacio propio, la sociedad neoliberal ha recuperado la necesidad de producirlo para asentar una identidad sustentada en el sentido común económico.

La identidad del grupo dominante en el neoliberalismo no se fundamentaría tanto en la renta, como en una idea de prestigio determinado por los conocimientos adquiridos, unos hábitos comunes y unas referencias espaciales que constituirían el capital cultural asociado a este grupo. Es dificilmente cuestionable que este es un reflejo de los modos de producción neoliberales, donde la expansión tecnológica ha hecho cambiar la percepción de la escala social y salarial. En cualquier caso, no debemos obviar que el neoliberalismo ha ido ocupando el lugar histórico, vinculando el pasado con la idea actual de prestigio, convirtiendo estructuras urbanas históricas en espacio de representación de sus valores. Al compartir estos espacios por el grupo social dominante en cuanto a las estructuras formales salariales y laborales, reforzaría su identidad y por tanto, sus valores se convertirían en hegemónicos en la ciudad actual.

En lo relativo a la ciudad de Madrid, la evolución del modelo de producción y su intensificación neoliberal nos deja una segmentación social fundamentada en una pequeña clase dirigente, heredera de alguna manera de los rentistas e industriales del siglo XIX, una clase media de relativa estabilidad laboral —en donde destacarían sectores de alta cualificación académica pero altamente precarizados-, y finalmente una importante clase servidora que ocuparía los trabajos menos remunerados, la mayor temporalidad laboral y la menor formación académica (Lacalle, 2006), formada, fundamentalmente, de inmigrantes, mujeres y jóvenes. Esta morfología social ha quedado evidenciada en los últimos años posteriores a la crisis económica de 2008 (Observatorio Metropolitano, 2007), y son patentes al analizar los datos que se van sucediendo como consecuencia de la crisis sanitaria actual ${ }^{2}$.

Este esquema morfológico responde a los modelos de producción del espacio desarrollados en las últimas décadas, tal y como ha resaltado Roch (Roch Peña, 2009) (26-30 de mayo de 2008) (2015), lo que ha supuesto la imposición del marco social sobre el espacio físico, mostrando una inter-relación entre forma urbana y formación social. Así, se habría llevado a cabo la depuración de un modelo de segregación socio-espacial a través de los procesos inmobiliarios llevados a cabo en las dos

\footnotetext{
${ }^{2}$ A este respecto conviene que se repasen los datos preliminares que se están obteniendo sobre cómo las causas y consecuencias de la morfología social de Madrid tienen sobre la pandemia. Estos datos pueden encontrarse en los trabajos de Córdoba Hernández y otros coautores (Córdoba et al, 2020a) (Córdoba et al, 2020b)
} 
décadas de expansión neoliberal. Los precios inmobiliarios habrían tendido a producir un espacio capaz de asignar identidades comunes que reproducirían la jerarquización morfológica existente. Tal y como afirma Méndez (2012), la solución espacial neoliberal pasaría por el protagonismo creciente de las inversiones inmobiliarias, concentrándose en las áreas más rentables y trasladando su presión en el sistema de precios.

El caso de Madrid refleja el tránsito de la complejidad social del centro urbano, al incremento de una jerarquización lograda a partir de la posición social de los individuos como condición espacial, y la producción de una imagen colectiva del espacio social dentro del proceso de asignación del valor inmobiliario, incluso su reproducción en el sistema de precios de los alquileres y su relación con el turismo

Desde los años 1960, Madrid creció a base de inversiones en espacio residencial, pero la transición hacia un modelo de propiedad universal impuso la necesidad de la inversión pública para financiar una expansión de las redes de infraestructuras y equipamiento urbano. Tras la aprobación del Plan General de Madrid en 1997, se desarrollan las grandes infraestructuras viarias, de abastecimiento y depuración de agua, gestión de residuos, suministro de energía (Gavira Golpe, 1999) o transporte y distribución de mercancías (Ruiz Sánchez, 1999), todas ellas financiadas casi en exclusiva con capital público. El papel de este queda subsidiario del capital privado, quien requiere de la solución infraestructural para sostener su crecimiento y reforzar la acumulación de capital económico en torno a la producción de ciudad.

Es en esta dinámica en el que las inversiones en actividades culturales se convierten en estrategia esencial para la depuración del modelo. De un lado refuerza el carácter de motor de la recuperación de espacios degradados, ya sean contenedores en desuso o áreas urbanas históricas. De otro lado, contribuyen a la expansión de valores asociados al prestigio y al ascenso social, consiguiendo con ello la identificación como grupo, de individuos con un estatus laboral y formativo similar. La intensidad de las inversiones llevadas a cabo entre los años 2000 y 2011 parecen corroborar esta idea, y la crisis de 2008 indicaría un punto de inflexión en un cambio de modelo que ya estaba en marcha.

De hecho, los cambios ocurridos en Madrid nos inducen a pensar que las instituciones culturales, a partir de la inversión en espacios urbanos y arquitectónicos, se sirven de una compleja interacción público-privada que permite la producción de una nueva simbología urbana, que a modo de marca (García Pérez, 2014) han condicionado estrategias de regeneración y recuperación urbana con efectos sobre el sistema de precios inmobiliarios. Esta estrategia habría permitido la atracción de pequeñas unidades de creación o expansión cultural —comercio artesanal, pequeñas galerías de arte, librerías, tiendas de diseño o moda, etc.- - pero también la atracción de visitantes. La transformación cultural ha permitido, pues, la atracción del turismo 
internacional, globalizando con ello la ciudad, interrelacionándola en competencia con otros espacios urbanos ${ }^{3}$.

Con esta percepción, se asocia, tal y como sostienen Lefebvre o Harvey, la imagen de arte y de las instituciones culturales como una especie de alter ego de la economía, que aporta a la reproducción social un ámbito más sublime y elevado que se superpone sobre la lógica materialista del mercado. La percepción del arte como estrategia del capital, asociada a la lógica mercantil de la mercancía, diluyendo con ello una percepción independiente de ambos ámbitos (Smith, 2010). Esta lógica mercantil de la cultura se complementaría con una transferencia de urbanidad que conllevaría la modificación de hábitos, costumbres, formas de vida o representaciones sociales, tal y como afirma Martínez (2018), y que culminaría en la intensificación de la ciudad como espacio del turismo, reajustando los parámetros urbanísticos y sociales del espacio urbano actual.

\section{Proceso de acumulación en el espacio de centralidad de Madrid: la Almendra Central}

Un análisis del espacio de centralidad podría confirmar las sospechas de que es en éste, el lugar donde se representa el ascenso social en la expansión neoliberal. En el caso de Madrid, podríamos ceñirlo a la evolución y a la construcción histórica, pero encontramos datos suficientes que asimilan el área funcional de la Almendra Central como espacio central de Madrid (Morcillo Álvarez, 2015).

La Almendra Central es el espacio comprendido en el interior del primer cinturón viario metropolitano, y acoge en su interior los ámbitos establecidos en el planeamiento como el centro de la ciudad: el Casco Antiguo y el Centro Histórico. Esta determinación no está definida en documentos de planeamiento, pero sí se recoge en numerosa bibliografía municipal, construyendo un espacio simbólico en torno a la idea de centro. Son llamativas las referencias en el Plan Especial de Revitalización del Centro (Oficina Centro, 2004) o en el Proyecto Madrid Centro (Ayuntamiento de Madrid, 2011), último documento de análisis y propuesta sobre el centro de Madrid. Es significativo que, pese a ello, la declaración del área Madrid Central —o la reciente modificación Madrid 360-, haya retornado a una delimitación que coincide en gran medida con el Casco Antiguo (recinto histórico de Madrid) y que las referencias a la Almendra Central hayan ido desapareciendo de la literatura municipal, ignorando el espacio simbólico producido y volviendo al recinto histórico como acumulador de capital.

\footnotetext{
${ }^{3}$ No conviene olvidar que muestra de esta competencia urbana son las tablas que reflejan la posición de cada ciudad respecto de una serie de indicadores urbanos. Entre ellos, la capacidad de atraer turismo internacional, la flexibilidad de su mercado laboral, la producción de capital, el número de empresas globales que alojan, etc., y que son los principales operadores inmobiliarios quienes las fomentan (Córdoba y Morcillo, 2020)
} 
Figura 2: Delimitación de la Almendra Central, el Casco Antiguo y el Centro Histórico en la dimensión de la ciudad de Madrid

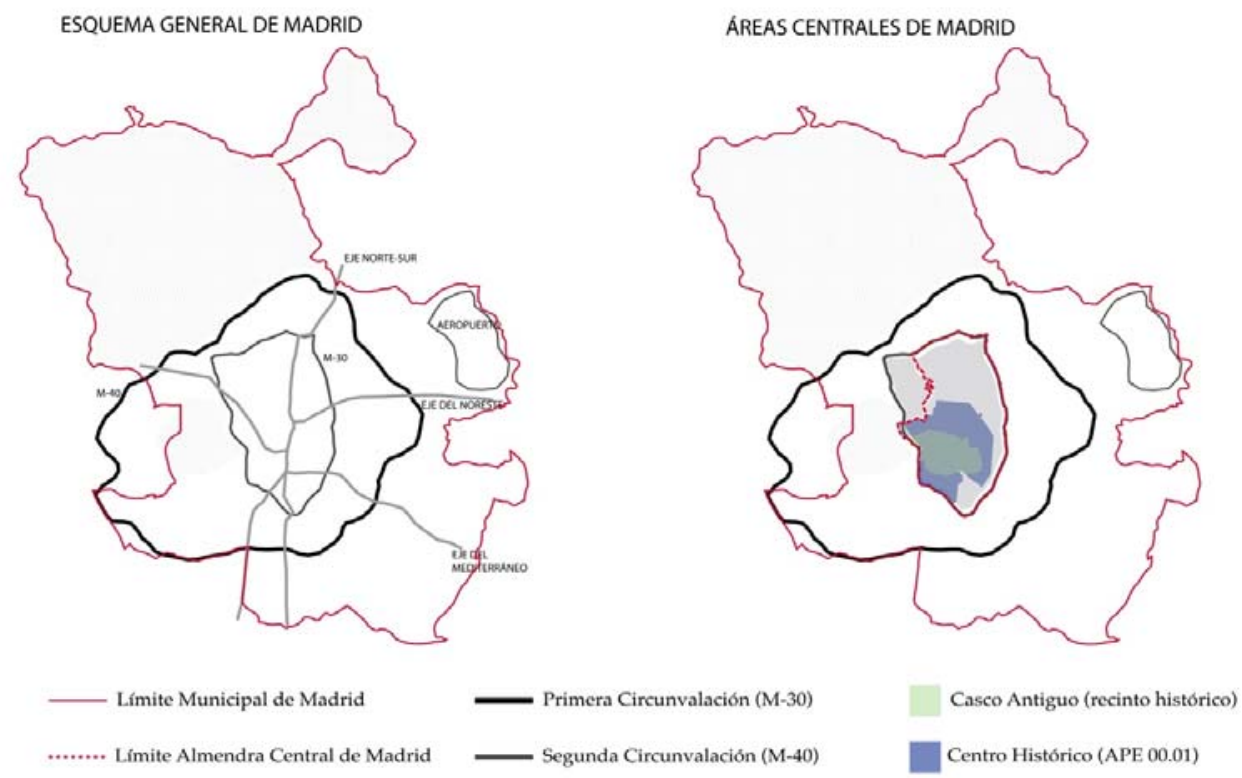

Fuente: Elaboración propia

A pesar de ello, la delimitación de la Almendra Central como espacio central insinúa la paulatina expansión de las características de centralidad propias del modelo, que han hecho de este recinto un espacio para la acumulación de capital en cualquiera de sus formas, imponiéndose sobre la estructura social históricamente definida.

En esta expansión ha sido necesaria una fuerte inversión de capital público que ha condicionado la transformación de la imagen y la estructura socio-espacial. A partir de 1998, se inicia un ciclo expansivo de la economía madrileña, llevándose a cabo numerosas inversiones en la Almendra Central (Morcillo Álvarez, 2015b) que han estado dirigidas a transformar la imagen del centro urbano y a redefinir la caracterización de la centralidad. Se realiza una expansión funcional de esta hasta los límites de la M-30, cuyo soterramiento parcial se ha convertido en la expresión más convincente de las pretensiones neoliberales, concentrando la inversión durante los años de mayor expansión. Así, se han sucedido desde 1998 inversiones que han girado en torno a la identificación del espacio central con el turismo y los negocios. De hecho, ambos usos han sido habitualmente solapados en una estrategia municipal por atraer inversiones y capital internacional a partir del turismo de negocios. Esto llevó al municipio a una fuerte competencia por atraer congresos, ferias y eventos de carácter internacional, fundamentalmente apuesta por alojar unos Juegos Olímpicos, y más recientemente, la celebración de eventos deportivos internacionales, el COOP25, 
o la pretensión de alojar el Mobile Congress aprovechando el avance de la pandemia de 2020.

Este solapamiento de usos ha permitido la expansión del área de centralidad para intentar ligar los espacios de producción con los históricos y de atracción turística, por lo que no debe extrañar que el reforzamiento del eje central de negocios tenga su continuidad formal y estructural con el eje cultural. El eje central de negocios podemos deducirlo de los trabajos de Morcillo (2015a) en torno a las actividades económicas y se definiría por la acumulación de actividades relacionadas con la empresa y los negocios. Por otro lado, las áreas culturales y los ejes culturales quedan determinados por los grandes contenedores y se determinan en torno al llamado Paseo del Arte (Morcillo Álvarez, 2017).

Figura 3: Ejes estructurantes de la Almendra Central: área de negocios y cultural. Año 2004.

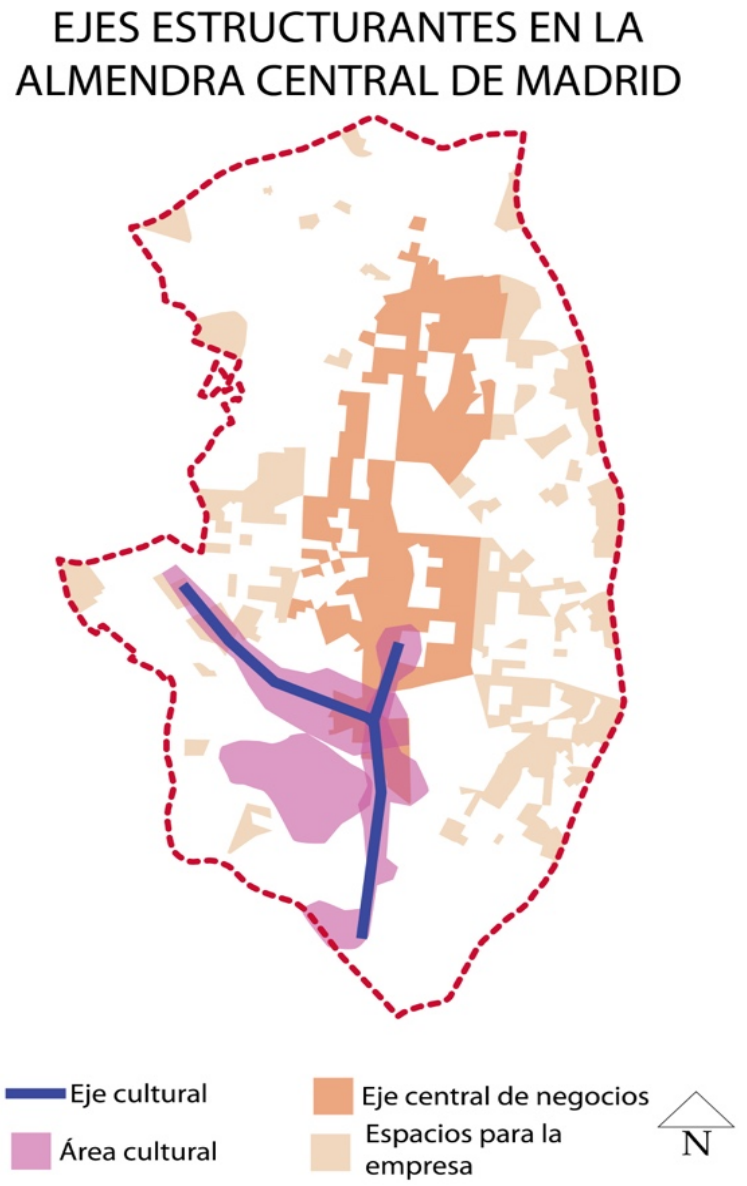

Fuente: Elaboración propia 
Las inversiones relacionadas directamente con la cultura parecen perseguir una valorización del espacio de centralidad para atraer nuevas fuentes de inversión relacionadas con el turismo y el consumo de ocio. Estas pretensiones quedan patentes no solo en la búsqueda de nuevos focos turísticos, sino también en el reforzamiento del turismo de negocios asociado al desarrollo del sector servicios y al aumento de ferias y congresos. Además, el resultado parece reflejar una imagen de la centralidad como identificador de posición social, de tal manera que lugares y contenedores arquitectónicos se han terminado convirtiendo en espacios para el consumo y la actividad económica.

\section{El uso cultural en la Almendra Central}

Para confirmar los argumentos esgrimidos, nos planteamos la necesidad de verificar los aspectos sucedidos y conocer los cambios ocurridos en las dos últimas décadas, para lo cual tomaremos como punto de partida la localización de los principales usos culturales en la Almendra Central (Subdirección General del Plan General, 2004). El estudio realizado por el Área de Urbanismo, apunta al Centro Histórico como un espacio de concentración de equipamientos culturales, donde proliferan cines, teatros o museos, es decir, aquellos lugares con capacidad de atracción de la población urbana y metropolitana. Esto supone la producción de un espacio simbólico de concentración de actividad que dará un salto cualitativo con la apertura del tren de cercanías en 2009 y las posteriores inversiones en la escena pública.

Observamos que no solo hay un claro desequilibrio entre el centro y la periferia en cuanto al uso del suelo, sino también en cuanto a los tipos y características de los equipamientos. De esta manera, la mayor parte de los equipamientos culturales de ámbito de ciudad, se encuentran localizados en la Almendra Central, mientras que en los distritos de la periferia se localizan equipamientos destinados al servicio a la población residente. En lo referente a los equipamientos privados, como podrían ser los cines o los teatros, el desequilibrio es incluso mayor, solo mitigado por la explosión de centros comerciales y de ocio en los límites urbanos o en los nuevos desarrollos urbanísticos.

En la Almendra Central este desequilibrio es aún más evidente, pues la capacidad de acumulación se concentra en el Centro Histórico y el Casco Antiguo, llevando al resto del espacio central a un gran vacío ocupado, fundamentalmente, por centros comerciales y de ocio. Aunque no hay grandes variaciones en la calificación del suelo, en el año 2005 se aprueba una modificación del planeamiento que permite la descalificación del uso cultural en el suelo de la ciudad, permitiendo la reconversión de espacios culturales en espacios comerciales o de otro tipo de ocio (Alcaide, 2015), dando muestras de un cambio de modelo en cuanto a las formas culturales de la ciudad y a la morfología de expansión. 
Figura 4: Usos culturales en la Almendra Central. Año 2004

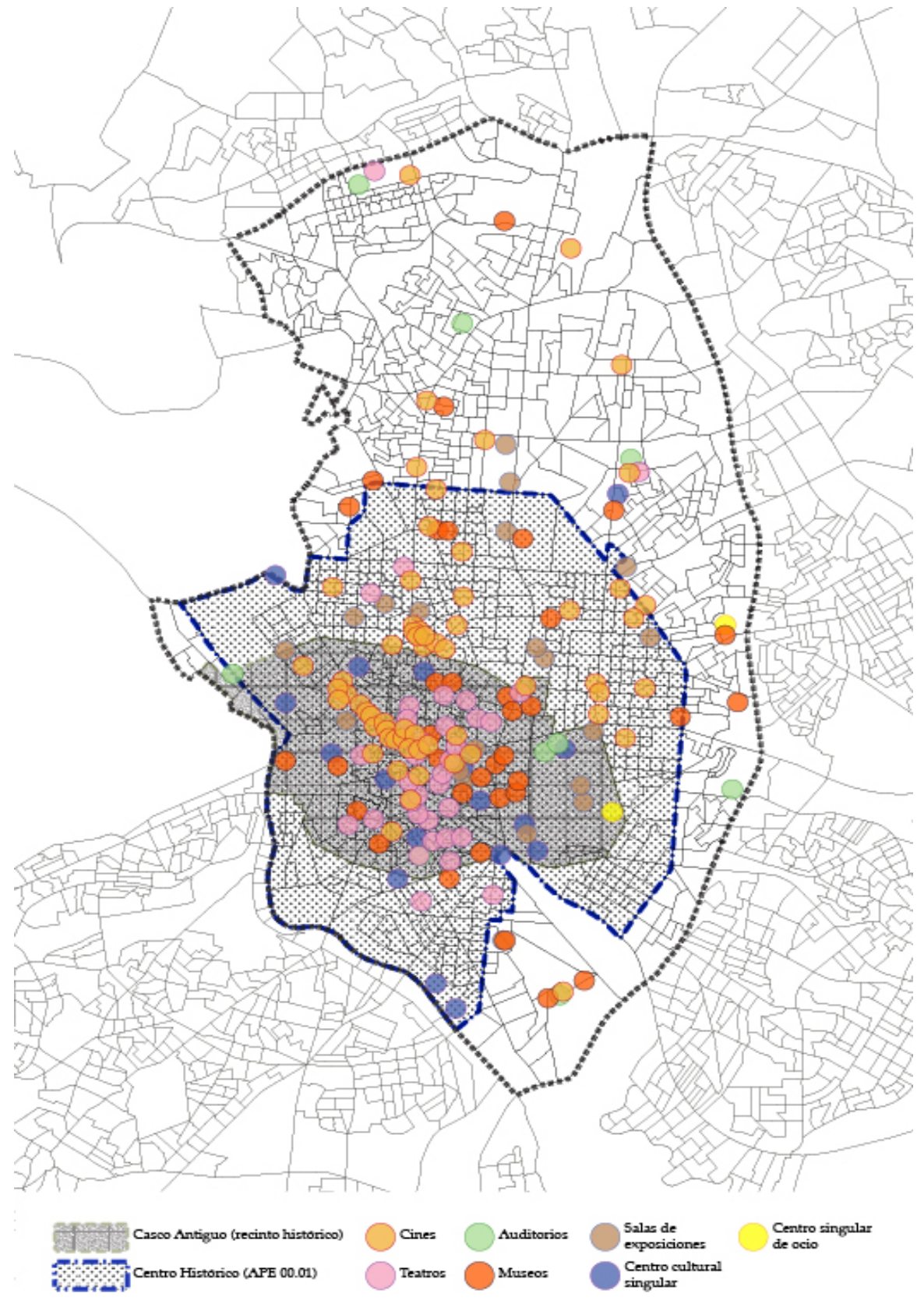

Fuente: Ayuntamiento de Madrid y elaboración propia. 
A través de los mapas de implantación de actividades culturales en la Almendra Central, obtenidos a partir de las series estadísticas del DUAE, podemos comprobar no solo el incremento del número de establecimientos, sino también su expansión en los límites de la Almendra Central, siendo este proceso especialmente intenso en el Centro Histórico.

Tabla 1: Usos culturales en la ciudad de Madrid. Año 2004

\begin{tabular}{|l|c|c|c|}
\hline & $\begin{array}{c}\text { ALMENDRA } \\
\text { CENTRAL }\end{array}$ & $\begin{array}{c}\text { RESTO DE LA } \\
\text { CIUDAD }\end{array}$ & TOTAL \\
\hline $\begin{array}{l}\text { Centros Culturales } \\
\text { Singulares }\end{array}$ & 25 & 4 & 29 \\
\hline Museos & 39 & 13 & 52 \\
\hline Salas de exposiciones & 20 & 1 & 21 \\
Sedes Institucionales & 19 & 4 & 23 \\
Centro singular de ocio & 5 & 6 & 11 \\
Teatros & 40 & 4 & 44 \\
Cines & 58 & 22 & 80 \\
Auditorios & 7 & 21 & 28 \\
\hline \multicolumn{1}{|c|}{ TOTALES } & $\mathbf{2 1 3}$ & $\mathbf{7 5}$ & $\mathbf{2 8 8}$ \\
\hline
\end{tabular}

Fuente: Elaboración propia a partir de datos del Ayuntamiento de Madrid

Varios factores podrían explicar a qué se debe este proceso expansivo de la actividad cultural, unos relacionados con el cambio de referencias sociales y otros relacionados con aspectos de atracción de capital y turismo. En cualquier caso, nos interesan los posibles cambios morfológicos producidos, pues la expansión de las actividades parece indicar una expansión de los valores de prestigio que otorga la centralidad al total de la Almendra Central. Esto se contrapone con la concentración de equipamiento cultural en el Casco Antiguo, aunque no necesariamente son aspectos contradictorios. En este caso, el tipo de equipamiento cultural responde a la lógica de zonificación propia de épocas pasadas, mientras que en el proceso de expansión se percibe un cambio de los cuadros sociales y que situaría a la cultura como nueva referencia en la definición de la posición social de los individuos. Su expansión por ámbitos como el eje Norte Sur y el río vendrían a reforzar esta idea.

\section{Inversiones culturales en la expansión de la centralidad}

En los años de expansión neoliberal las inversiones en infraestructuras culturales parecen haber intensificado la competencia espacial con la que se escenificaba la pugna por la identificación de la posición social en el espacio urbano. De alguna manera, han contribuido a este proceso a partir de la recuperación de espacios 
degradados, edificios en desuso o infrautilizados, con la que se ha incrementado la acumulación de capital cultural y simbólico en la Almendra Central, superponiendo cultura y economía.

Ya en la década de los años 2000, eclosiona el proceso de reforzamiento simbólico de la cultura como factor de posición social, aunque su traslado al espacio físico de la Almendra Central se inicia en épocas anteriores. El Plan General de Madrid de 1997 recoge diferentes actuaciones estructurantes, pero ninguna de ellas se centra específicamente en el ámbito cultural. Sin embargo, entre sus objetivos está posicionar a Madrid en un circuito financiero europeo, atrayéndolo a partir del turismo, lo que hace potenciar el principal eje museístico. Todos los esfuerzos estructurantes de Madrid se centran en grandes operaciones para llevar a Madrid al límite de su capacidad (de Santiago Rodríguez, 2012) en cuanto a la urbanización del suelo, siendo a partir del año 2003 cuando la cultura se convierte en un argumento de intervención urbanística, seguramente por su capacidad para transformar espacios degradados en la ciudad histórica, pero también por su facultad para expandir las características y valores de centralidad. Así, son sucesivos documentos municipales los que empiezan a valorar la Almendra Central como el centro de Madrid, consiguiendo un cambio en el discurso, y en donde la definición de diferentes ejes simbólicos de carácter cultural contribuye al cambio de los cuadros simbólicos. Esto supone la acumulación de recursos - económicos, humanos y creativos - en torno a grandes contenedores arquitectónicos, y la producción de una red de espacios de uso administrativo-cultural vinculados directa o indirectamente con los espacios históricos, dando pie a una relación simbólica entre cultura y centralidad.

Esta relevancia de la cultura en la expansión de la centralidad de Madrid eclosiona en el Plan Centro que es aprobado en el año 2011, en donde se manifiesta:

«el área central de Madrid presenta un importante tejido cultural, un vivero de creadores cada vez más rico en cantidad y calidad, que necesita mayores facilidades para instalarse en la ciudad y para contar con espacios adaptados a sus necesidades» (Ayuntamiento de Madrid, 2011)

Sin duda, la profusa documentación municipal en este tema nos lleva a pensar que hay una causalidad entre cultura y morfología, que lleva a considerar a la primera un elemento estructurante de la ciudad, para lo cual se requerirían no solo de espacios destinados a actividad museística con las que atraer turismo, sino también espacios de creación vinculados a las artes plásticas, escénicas o del diseño. Esto ha supuesto que las intervenciones puntuales sirvieran a la estrategia de poner grandes espacios de Madrid a disposición del nuevo modelo de acumulación de capital, donde lo simbólico determina las relaciones económicas y sociales.

Es así como a lo largo de dos décadas se han ido desarrollando numerosos equipamientos, como bibliotecas, centros culturales y cívicos, museos o salas de exposiciones, con la pretensión de solventar el desequilibrio territorial existente entre las áreas centrales y el resto de la ciudad. Aunque en el Plan General se recoge la recuperación del eje museístico Recoletos-Prado, en general, estas actuaciones responden a planteamientos posteriores, impuestos por una lógica neoliberal alejada de la planificación urbanística. Así es como debemos entender la importante inversión 
que ha terminado configurando una serie de ejes culturales que amplían los existentes pero que aún no cuentan con una definición ni estructura desde el planeamiento formal.

Así ha ocurrido con el Eje Recoletos-Prado. Configurado desde el planeamiento como un eje museístico para reforzar la atracción del turismo, ha sido reconfigurado con la recuperación de grandes contenedores "industriales", como la Serrería Belga, el Matadero y el Palacio de Telecomunicaciones y Correos. Este nuevo eje virtual, cuya extensión hasta el río queda apoyada por la aparición de operadores culturales en su ámbito sur $^{4}$, se configura como un gran eje simbólico que se extiende desde el centro histórico hasta los límites de la nueva configuración de la centralidad.

Tabla 2: Inversión pública en infraestructuras culturales.

\begin{tabular}{|l|c|c|c|}
\hline \multicolumn{1}{|c|}{ PROYECTO } & APROB. & EJECUCIÓN & PPTO. \\
\hline MATADERO MADRID & $\mathbf{2 0 0 3}$ & $\mathbf{2 0 1 1}$ & $\mathbf{1 8 . 7 7 5 . 0 9 2 , 0 0 €}$ \\
\hline INTERMEDIAE / SERRERÍA BELGA & $\mathbf{2 0 0 5}$ & $\mathbf{2 0 0 7}$ & $\mathbf{1 . 4 3 2 . 3 0 0 , 0 0} €$ \\
\hline REMODELACIÓN CONDE DUQUE & $\mathbf{2 0 0 4}$ & $\mathbf{2 0 0 8}$ & $\mathbf{1 0 . 0 6 2 . 6 2 9 , 0 0 €}$ \\
\hline REMODELACIÓN PALACIO DE CORREOS & $\mathbf{2 0 0 6}$ & $\mathbf{2 0 1 1}$ & $\mathbf{2 0 . 9 6 3 . 1 7 8 , 0 0 €}$ \\
\hline CASAS NACIONALES & & & $\mathbf{1 0 . 8 0 0 . 0 0 0 , 0 0 €}$ \\
\hline CASA SEFARAD-ISRAEL (PALACIO DE CAÑETE) & 2009 & 2010 & $6.900 .000,00 €$ \\
\hline CASA ÁRABE (ESCUELAS AGUIRRE) & 2006 & 2008 & $3.900 .000,00 €$ \\
\hline
\end{tabular}

Fuente: Elaboración propia a partir de datos del Ayuntamiento de Madrid

El principal proyecto cultural emprendido entre los años 2000 y 2011 es el del Matadero Madrid. La inversión ha permitido la recuperación de un complejo industrial del siglo XIX adaptando las naves y edificios destinados a matadero municipal a diferentes usos culturales y creativos. En la propia descripción de la actual instalación cultural (Matadero Madrid, 2011), se significa por ser un espacio de creación para la difusión cultural, convirtiéndose en un lugar determinante en la producción y expansión de referencias urbanas.

En este espacio de producción y consumo de cultura se alojan numerosas instituciones públicas, pero también privadas, relacionadas con diferentes campos del diseño, la moda y otros ámbitos, con el objetivo estratégico de convertir Matadero Madrid en el principal centro de producción de capital cultural en Madrid. Muestra de esta estrategia son las instituciones alojadas en sus instalaciones:

\footnotetext{
${ }^{4}$ Este sería el caso de la Casa Encendida (edificio que aloja la sede cultural de la Fundación Bankia), el Circo Price o la Tabacalera (antiguo edificio de la Fábrica de Tabacos, hoy en día gestionado por el Ministerio de Cultura).
} 
Tabla 3: Instituciones culturales implantadas en Matadero Madrid ${ }^{5}$.

\begin{tabular}{|c|c|c|c|}
\hline & $\begin{array}{l}\text { NOMBRE DE LA } \\
\text { INSTITUCIÓN }\end{array}$ & OBJETIVOS & $\begin{array}{c}\text { FECHA DE } \\
\text { IMPLANTACIÓN }\end{array}$ \\
\hline \multirow{4}{*}{ 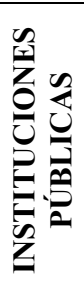 } & Intermediae & $\begin{array}{l}\text { Mediación con la comunidad del entorno } \\
\text { del centro }\end{array}$ & 2007 \\
\hline & Naves Matadero & $\begin{array}{l}\text { Creación y difusión de nuevos lenguajes } \\
\text { escénicos para conectar a creadores con la } \\
\text { ciudadanía. }\end{array}$ & 2015 \\
\hline & $\begin{array}{l}\text { Oficina de } \\
\text { Coordinación }\end{array}$ & $\begin{array}{l}\text { Exposiciones en múltiples categorías } \\
\text { artísticas }\end{array}$ & 2008 \\
\hline & Cineteca & Difusión cinematográfica. & 2008 \\
\hline \multirow{5}{*}{ 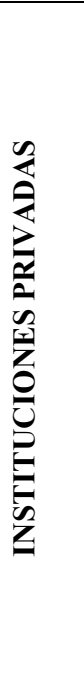 } & Central de Diseño & $\begin{array}{l}\text { Promoción del diseño. } \\
\text { Gestionado por la Fundación Diseño de } \\
\text { Madrid (DIMAD) }\end{array}$ & 2007 \\
\hline & Extensión AVAM & $\begin{array}{l}\text { Fomento de la participación, el debate y el } \\
\text { intercambio entre artistas. } \\
\text { Gestionado por Artistas Visuales } \\
\text { Asociados de Madrid (AVAM) }\end{array}$ & 2011 \\
\hline & $\begin{array}{l}\text { Centro de } \\
\text { residencias artísticas }\end{array}$ & $\begin{array}{l}\text { Estímulo de la producción artística a partir } \\
\text { de la movilidad de creadores y la } \\
\text { generación de entornos colectivos de } \\
\text { aprendizaje. }\end{array}$ & 2011 \\
\hline & Casa del Lector & $\begin{array}{l}\text { Promoción de la lectura y el mundo del } \\
\text { libro. } \\
\text { Gestionado por la Fundación German } \\
\text { Sánchez. }\end{array}$ & 2012 \\
\hline & Factoría Cultural & $\begin{array}{l}\text { Apoyo al Emprendimiento de las } \\
\text { Industrias Culturales y Creativa. } \\
\text { Gestionada por el Vivero de Industrias } \\
\text { Creativas. }\end{array}$ & 2014 \\
\hline
\end{tabular}

Fuente: Matadero Madrid

El otro polo de este eje cultural tiene como referencia el antiguo edificio de Correos, construido a principios del siglo XX como Palacio de Telecomunicaciones pero que había ido perdiendo su actividad según se iba transformando el sistema de correos. La recuperación de este edificio como símbolo de la nueva centralidad de Madrid se realizó a partir de una compleja operación inmobiliaria, consistente en la permuta de edificios entre administraciones y entidades financieras, de tal manera que en ella intervinieron, entre 2004 y 2006 los Ministerios de Hacienda, Fomento y

\footnotetext{
${ }^{5}$ Es importante destacar que el listado de instituciones implantadas en Matadero (Matadero Madrid, 2011) es cambiante, en función de la visión y gestión de cada equipo de gobierno. Así, desde su apertura también han tenido espacio instituciones como Red Bull España que mantuvo abierta la Nave de Música, con residencias artísticas, o las Naves del Español, como espacio satélite del Teatro Español.
} 
Cultura, el Ayuntamiento de Madrid y el Banco Santander. La operación tenía como finalidad recuperar una serie de edificios en desuso o infrautilizados, de tal manera que se dotara al Ayuntamiento de Madrid de un edificio donde localizar una nueva sede y al Tribunal Constitucional que ocupara la suya en el centro de Madrid, dotándole del prestigio que esta institución demandaba.

Por sí solo, este juego de permutas da muestra de la relevancia que para todas las instituciones involucradas jugaba el espacio central. La operación finaliza convirtiendo la Sede del Banco Santander en sede del Instituto Cervantes, lo que supuso que el centro de Madrid ganara una nueva sede cultural, ampliando su representatividad para la atracción de turismo y reforzando el eje cultural. En el caso del Palacio de Telecomunicaciones, la inversión realizada fue destinada a la adaptación del edificio para centro de divulgación cultural, centro de atención turística, dependencias administrativas y sede del Ayuntamiento de Madrid.

Este último uso supuso trasladar la histórica sede de su ubicación original hasta el centro de un eje cultural y simbólico que pasará, seguramente, a convertirse en el principal espacio de representación de una nueva ciudad que aspiraba, por entonces, a convertirse en el centro financiero del sur de Europa (Córdoba y Morcillo, 2020) . $^{6}$ Vinculada la sede del Ayuntamiento, con los principales centros museísticos, que atraen la mayor cantidad de turistas que visitan Madrid, el antiguo Palacio de Telecomunicaciones pasaría a convertirse en un espacio para la recepción de los turistas, con la intencionalidad de ser el primer contacto de la ciudad con sus visitantes a través de un sistema de información turística y cultural.

Como decíamos, a lo largo de este eje se localizan otra serie de usos culturales que refuerzan el carácter singular y representativo y que han permitido la transformación de los espacios populares de su entorno, reconvirtiendo su uso, con fuerte presencia industrial, en un espacio para la creación, pero también para el consumo de ocio, tal y como nos muestran los cambios de actividad sucedidos desde el entorno de la estación de Atocha hasta los límites de Matadero Madrid.

\footnotetext{
${ }^{6}$ Esta vieja aspiración, presente en los documentos municipales desde el Plan General, y defendida intensamente por el gobierno local en el período de 2003-2011, ha sido recientemente retomada por los gobiernos local y regional (Maharg-Bravo, 2016) (G. Treceño, 2016) a raíz del llamado "Brexit". Éste ha hecho que Madrid volviera a aspirar a esta condición de gestor del capitalismo en el sur de Europa.
} 
Figura 5: Evolución del número de establecimientos culturales en la Almendra Central. Año 1998 (sup. izq), 2004 (sup. dcha.) y 2010 (inf.).

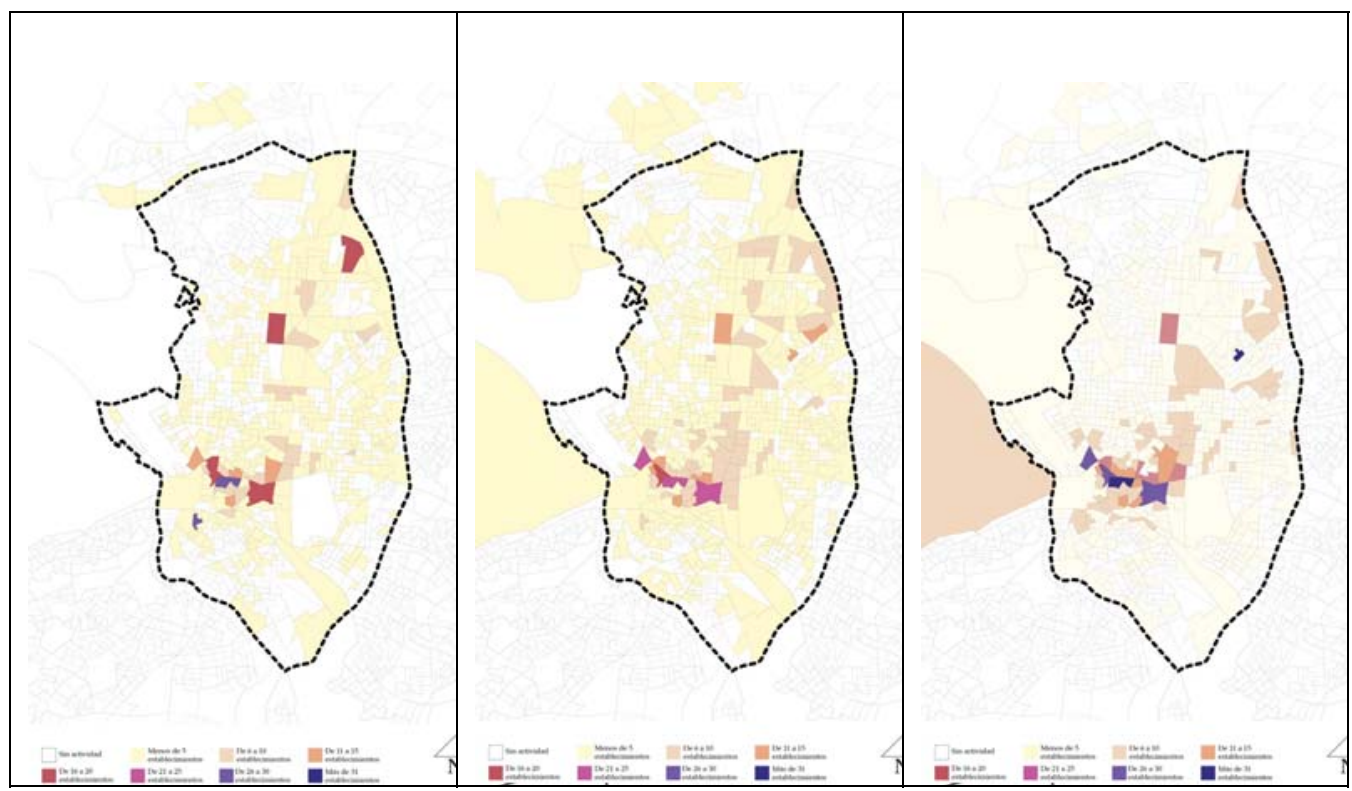

Fuente: Elaboración propia a partir de datos del DUAE.

Sin duda alguna, el eje Cibeles-Río es el más representativo en la ciudad y es el que mejor muestra la producción del espacio de exclusividad de la Almendra Central a partir de la cultura, pero a través de otras operaciones se están reforzando nuevos polos de actividad donde se solapan los valores culturales con los comerciales y turísticos. La implantación del espacio del Conde Duque y las Casas Culturales internacionales, contribuirían a este hecho, tras la realización de fuertes inversiones que han permitido recuperar edificios en desuso y revitalizar sus entornos urbanos.

Es el caso del antiguo Real Cuartel de Guardias de Corps, construido en el siglo XVIII y que mantuvo su actividad hasta principios del siglo XX, momento en el que empezó su abandono, contribuyendo con ello a la degradación de su entorno. En los años 1980, el Ayuntamiento de Madrid adquiere el cuartel y se inicia un proceso de recuperación del edificio como Biblioteca Municipal y Archivo de la Ciudad. Aunque implantados estos usos, la recuperación del edificio es lenta por el alto coste y las malas condiciones económicas del momento. En el año 2005 se aprueba un Plan Director que aprovecha el ciclo económico y supone que, entre 2005 y 2011, se lleven a cabo grandes inversiones. Esto ha supuesto la recuperación del edificio y su puesta en servicio como una gran infraestructura cultural que ha contribuido a la recuperación de la escena urbana, pero que también ha servido de atracción de actividades de ocio y consumo. 
Siguiendo una lógica de exclusividad, parecen contribuir a un proceso de elitización del ámbito ${ }^{7}$, que podría facilitar la construcción de un importante polo de atracción turística, comercial y de ocio en uno de los extremos de la Gran Vía, produciendo un importante contrapeso representativo y simbólico al eje cultural del Paseo del Prado, contraponiendo el turismo cultural frente al turismo comercial.

Finalmente, las Casas Culturales internacionales han seguido la lógica de recuperar edificios de propiedad municipal y de alto valor histórico, para convertirlos en centros de intercambio cultural con dependencias expositivas, institucionales y de difusión cultural de las regiones de referencia. La pionera fue la Casa de América, localizada en la Plaza de Cibeles, y cuya adquisición por el Ministerio de Exteriores permitió recuperar uno de los edificios más característicos de la primera expansión de Madrid en el siglo XIX. Desde su inauguración en 1992, Casa América se ha convertido en un centro de relación e intercambio entre los diferentes países latinoamericanos. En la actualidad juega un papel urbano relevante, pues localizada junto al edificio Centro-Centro, refuerza con su actividad el eje Recoletos-Río .

A partir de 2003, se inicia una nueva fase de internacionalización de Madrid y bajo la lógica de expansión de las relaciones culturales entre regiones con una historia común, es en la que siguen las otras tres instituciones de características similares. Se trata de las Casas Asia, Árabe y Sefarad, estas dos últimas con sede en Madrid fruto de los convenios firmados por el Ayuntamiento de Madrid y el Ministerio de Exteriores.

En lo referente a la Casa Árabe, la recuperación de las Escuelas Aguirre ha contribuido a la expansión de la influencia del eje cultural del Paseo del Prado hacia el Este, de tal suerte que facilitaría la construcción de un eje de carácter comercial cuya consolidación en las dos últimas décadas demostraría la expansión de valores de centralidad hacia los límites de la Almendra Central. Una muestra es la importante inversión realizada en esta zona con el objetivo de reforzar su carácter comercial de lujo, donde la operación de la calle Serrano es la más representativa.

La Casa Sefarad, situada en el Palacio de Cañete del complejo municipal de la Plaza de la Villa, también es un buen ejemplo de la recuperación del Patrimonio para la reproducción de capital cultural. Localizado en un ámbito donde la actividad administrativa es la más abundante, a lo largo de la última década se ha buscado la consolidación de este espacio como centro de turismo al tratarse de los orígenes de Madrid como ciudad, y por tanto como un fuerte espacio de acumulación simbólica.

Las Casas de América, Árabe y Sefarad son una muestra de la búsqueda que, a lo largo de dos décadas, se ha llevado a cabo en Madrid para reforzar aquellos aspectos que mejoren sus ventajas competitivas en el circuito financiero internacional. Con este tipo de instituciones no solo se ha contribuido al proceso de elitización de la

\footnotetext{
${ }^{7}$ Para un desarrollo mayor, pueden consultarse los textos de Morcillo (Morcillo, 2015b) (2014) respecto del tipo de actividad del centro urbano de Madrid y la influencia que éstas tienen en la normalización social que se ha llevado a cabo en las dos décadas de expansión neoliberal.
} 
centralidad a partir de la cultura, sino que también ha servido para atraer capital internacional de estas regiones, evidenciando, de nuevo, el solapamiento entre cultura y economía en el actual sistema de acumulación.

\section{Conclusiones}

Los años en los que en Madrid se ha producido la última gran expansión urbanística han coincidido con la intensificación de la inversión en infraestructuras culturales, mostrándose como dos elementos dentro de la misma estrategia de producción de capital. Aunque esta inversión no ha sido comparable en términos absolutos con las fuertes inversiones de capital en las áreas de crecimiento de Madrid, sí ha supuesto, en términos relativos una fuerte acumulación de capital económico y social en las áreas centrales de la ciudad. La concentración de la inversión en estos ámbitos parece responder a una lógica planteada por autores como Neil Smith (2005) o Neil Brenner (2009) de re escalamiento urbano que facilite los flujos de movimiento de capital, mercancías y personas a escala global, dando lugar con ello a una fuerte competencia espacial por posicionarse adecuadamente dentro de los espacios más rentables de estos flujos.

Los recientes procesos de turistificación a los que estamos asistiendo y que forman parte del más reciente debate teórico, académico y social, no pueden ser explicados adecuadamente sin este proceso de acumulación de capital vividos en las décadas precedentes. Inversiones públicas y privadas han contribuido a la producción simbólica de una imagen de Madrid, como centro de ocio y cultura. La acumulación simbólica y económica ha facilitado la creación de un gran eje cultural que parece poner de relieve el interés de utilizar la cultura como expansión de valores neoliberales, realizando una transformación de costumbres y formas de vida hacia la producción de nuevos cuadros de representación social. El espacio urbano se amoldaría, bajo esta perspectiva, a las nuevas características morfológicas, dando lugar a una producción espacial en base a las transferencias materiales y urbanísticas que condicionan el nuevo modelo de cultura.

Las infraestructuras culturales producidas o transformadas en los últimos años de la expansión inmobiliaria en Madrid han puesto en evidencia la vinculación entre cultura y centralidad, concentrando la mayor parte de ellas en espacios centrales y creando un gran eje cultural que tiene como polos de atracción grandes contenedores culturales para los que han sido necesarias fuertes inversiones públicas. Estas, junto a la interacción del sector privado, han sido elementos fundamentales en una estrategia de atracción del turismo que se ha desarrollado en Madrid en las dos últimas décadas.

Esta estrategia parece haberse impuesto sobre las estrategias urbanísticas, amoldando la estructura urbana a las necesidades de las relaciones de producción y facilitando una transferencia de valores que otorgan valor patrimonial a arquitecturas capaces de atraer capital económico. Curiosamente, ha sido la fuerte inversión en capital cultural y social la que ha permitido la consolidación de un modelo urbano inmobiliario, caracterizado por la fuerte segregación espacial y social, dando lugar a 
discontinuidades urbanísticas, pero sobre todo a la dificultad de acceso a la cultura de grandes sectores sociales.

Esto se debe no solo a los procesos de privatización vividos, sino también a la transformación de las referencias de clase en torno a la cultura, vinculando esta con los espacios más exclusivos de la ciudad, aspecto que limita, si no impide, el acceso de grandes sectores sociales, desvinculados de la ciudad y el centro urbano, históricamente construidos en base a la reproducción social y a los ámbitos de cotidianidad. Con el cambio de los cuadros sociales de representación, los espacios centrales quedan vinculados con la mercantilización del consumo, y, por tanto, su acceso solo es posible a aquellos sectores cuyas capacidades y características permitan entrar en el círculo de la mercantilización del espacio y de las formas de vida. Con ello, el gran eje central cultural, quedaría inaccesible para amplios sectores sociales de la ciudad, mientras que las facilidades de acceso físico y de movilidad, facilitan la atracción del turismo. La producción de espacio, tal y como preveía Lefebvre, se convierte en el principal argumento para la acumulación de capital, convirtiendo los procesos urbanísticos en facilitadores de la acumulación de capital económico.

Así es cómo la transformación urbana se ha llevado a cabo a partir de la reutilización de la estructura existente, a través de la modificación de usos, conceptos e imagen, construidos a lo largo de los años de expansión urbanística y los posteriores de crisis de acumulación. La recuperación del centro urbano dejaría de ser, pues, la consecuencia de la mejora de las condiciones de vida, y se convertiría en el nuevo instrumento para el cambio de las relaciones de producción y de las formas de vida.

\section{Referencias bibliográficas}

Alcaide, S. (7 de Enero de 2015). Los cines de Madrid podrán cambiar de uso para convertirse en pisos u oficinas.

El País(https://elpais.com/diario/2005/01/07/madrid/1105100654_850215.html).

Ayuntamiento de Madrid. (Enero de 2011). Área de Gobierno de Urbanismo e Infraestructuras. Recuperado el enero de 2014, de Proyecto Madrid Centro: http://www.madrid.es/portales/munimadrid/es/Inicio/Ayuntamiento/Urbanismo-eInfraestructuras/Informacion-Urbanistica/Proyecto-MadridCentro?vgnextfmt $=$ default\&vgnextoid $=0 \mathrm{~b} 65488 \mathrm{f} 7 \mathrm{c} 742310 \mathrm{VgnVCM} 1000000 \mathrm{~b} 205 \mathrm{a} 0 \mathrm{aRC}$ RD\&vgnextchannel $=44 \mathrm{f} 00 \mathrm{dd} 3 \mathrm{c} 84 \mathrm{fe} 110 \mathrm{VgnVCM} 2000000 \mathrm{c} 20$

Bourdieu, P. (2003). Las estructuras sociales de la economía. Barcelona: Anagrama.

Brenner, N. (2009). Restructuring, rescaling and the urban question. Critical Planning.

Cordoba Hernandez, R. y Morcillo Alvarez, D. (2020). Marco territorial de la producción de espacio en la región funcional de Madrid. Ciudades, DOI: https://doi.org/10.24197/ciudades.23.2020.71-93(23), 71-93.

Cordoba Hernandez, R., Fernandez Ramirez, C., Gomez Gimenez, J., Hernandez Aja, A. y Sanchez-Toscano Salgado, G. (2020a). Áreas Urbanas frente a Barrios. Análisis de las características urbanas ante el reto de la ciudad post-COVID19: el caso de Madrid. Ciudad 
y Territorio Estudios Territoriales(https://doi.org/10.37230/CyTET.2020.205.13.2), 665684.

Cordoba Hernandez, R., Hernandez Aja, A., Fernandez Ramirez, C. y Alvarez del Valle, L. (2020b). Hacia la Ciudad de los 15 minutos frente al COVID19. La densidad espacial de Madrid. Obtenido de Re-Hab. Crisis Urbana, Rehabilitación y Regeneración: http://vps181.cesvima.upm.es/re-hab/2020/05/06/hacia-la-ciudad-de-los-15-minutosfrente-al-covid19-la-densidad-espacial-de-madrid/

de Santiago Rodríguez, E. (2012). Una lectura de las políticas de suelo y los modelos urbanísticos madrileños desde mediados de los años 1990: de la liberalización a la resaca inmobiliaria. Geopolítica(s). Revista de estudios sobre espacio y poder, 3(1), 83-116.

Dirección General de Planificación Estratégica. Área de Gobierno de Desarrollo Urbano Sostenible. Ayuntamiento de Madrid. (2018). Madrid Recupera. Madrid: Ayuntamiento de Madrid.

G. Treceño, J. (23 de Noviembre de 2016). Cristina Cifuentes y Manuela Carmena unen esfuerzos para atraer inversión de la City tras el 'Brexit'. El Mundo.

García Pérez, E. (2014). Gentrificación en Madrid: de la burbuja a la crisis. Revista de Geografía Norte Grande(58), 71-91.

Gavira Golpe, C. (1999). Las redes de la capital en la capital de las redes. En R. LOPEZ DE LUCIO, Madrid 1979-1999. La transformación de la ciudad en veinte años de ayuntamientos democráticos. Gerencia Municipal de Urbanismo de Madrid.

Harvey, D. (2013). Ciudades Rebeldes. Del derecho de la ciudad a la revolución urbana (1 ${ }^{\mathrm{a}}$ edición ed.). Madrid: Akal.

Lacalle, D. (2006). La clase obrera en España. Madrid: El Viejo Topo / Fundación de Investigaciones Marxistas.

Maharg-Bravo, F. (19 de Diciembre de 2016). El 'brexit' podría iluminar Madrid. CincoDías.

Martínez Gutiérrez, E. (2018). Reflexividad urbana y mirada turística: sobre la producción y el consumo de los espacios urbanos. OBETS. Revista de Ciencias Sociales, Extra 1(13), $355-$ 381.

Matadero Madrid. (2011). Matadero Madrid. Recuperado el Enero de 2019, de http://www.mataderomadrid.org/index.php

Méndez, R. (2012). Globalización: neoliberalismo y dinámicas metropolitanas en Madrid. DAAPGE(19), 29-49.

Morcillo Álvarez, D. (2014). La Almendra Central de Madrid, espacio de normalización social. En Q. BONASTRA, M. VASCONCELOS P. JUNIOR, \& M. TAPIA (Ed.), XIII Coloquio Internacional de Geocrítica: El control del espacio y los espacios de control. Barcelona: Universidad de Barcelona.

Morcillo Álvarez, D. (2015a). Inversión, infraestructuras e imagen en la producción del espacio de centralidad en Madrid. Ciudades(18), 163-181.

Morcillo Álvarez, D. (2015b). La centralidad y la representación de la cotidianidad en la ciudad globalizada. Madrid y la evolución de la Almendra Central de 1998 a 2008. Tesis Doctoral (inédita). Madrid.

Morcillo Álvarez, D. (2017). La imagen en el cuerpo urbano: la transformación de Madrid hacia el consumo. Revista Urbano(35), 32-43. 
Observatorio Metropolitano. (2007). Madrid ¿La suma de todos? Globalización, territorio, desigualdad. Madrid: Traficantes de sueños.

Oficina Centro. Área de Gobierno de Urbanismo, Vivienda e Infraestructuras. (2004). Plan Especial de Revitalización del Centro Urbano. Madrid: Ayuntamiento de Madrid.

Roch Peña, F. (2015). Revolución neoliberal y "utopía" ciudadana, una batalla inaplazable. (I. U. Valladolid, Ed.) Ciudades(18), 49-68.

Roch Peña, F. (26-30 de mayo de 2008). La deriva patológica del espacio social en el modelo inmobiliario neoliberal madrileño. X Coloquio Internacional de Geocrítica. Barcelona: Universidad de Barcelona.

Roch Peña, F. (2009). Detrioro urbano y precio de la vivienda en Madrid. Ciudades(12), 171196.

Ruiz Sánchez, J. (1999). La transformación de la infraestructura de transporte. En R. LOPEZ DE LUCIO, Madrid 1979-1999. La transformación de la ciudad en veinte años de ayuntamientos democráticos. Madrid: Gerencia Municipal de Urbanismo de Madrid.

Sotelo Pérez, I., Sotelo Pérez, M. y Sotelo Navalpotro, J.A. (2019). Política económica y medioambiente: una aproximación. Observatorio medioambiental, 22, 91-109

Smith, N. (2005). El redimensionamiento de las ciudades: la globalización y el urbanismo neoliberal. En N. SMITH, \& D. HARVEY, Capital financiero, propiedad inmobiliaria y cultura. Barcelona: Contratextos.

Smith, N. (2010). ¿Son los museos tan solo un vehículo al servicio del desarrollo inmobiliario? En VVAA, Ideas Recibidas. Un vocabulario para la cultura artística contemporánea (págs. 130-149). Barcelona: Ensayo.

Subdirección General del Plan GeneraL. Área de Gobierno de Urbanismo e Infraestructuras. Ayuntamiento de Madrid. (2004). Distribución espacial del equipamiento cultural en el término municipal de Madrid. Ayuntamiento de Madrid, Subdirección General del Plan General. Madrid: Autor.

Subdirección General de Análisis Socioeconómico. (Enero de 2018). Ranking de ciudades europeas 2017. Barómetro de Economía de la Ciudad de Madrid, 55, 83-112. 